\title{
Form Birefringence Effect of Ultradrawn Polyethylene Determined by Two Dimensional Composite Structural Model
}

\author{
Masaru Matsuo ${ }^{\dagger}$ and Tetsuya OGita* \\ Department of Clothing Science, Faculty of Home Economics, \\ Nara Women's University, Nara 630, Japan \\ * Department of Materials Science and Engineering, Faculty of Engineering, \\ Yamagata University, Yonezawa 992, Japan
}

(Received October 13, 1990)

\begin{abstract}
Birefringence was calculated as a function of the content of voids, $\phi$, assuming that the morphology of ultradrawn polyethylene film is represented by a composite model. When birefringence is calculated using a parallel model, it is beyond $60 \times 10^{-3}$ at $\phi \geqq 0.24$. This indicates that the birefringence $>60 \times 10^{-3}$ observed for ultradrawn polyethylene films is attributed to the contribution of form birefringence. The theoretical calculation indicates that the real birefringence without contribution from form birefringence cannot be obtained by Wiener's method, since the maximum or minimum point of birefringence curve against refractive index of swelling liquid is not equal to the real birefringence.

KEY WORDS Content of Voids / Composite Model / Ultradrawn

Polyethylene / Form Birefringence / Swelling Liquid /
\end{abstract}

Since 1974, the preparation of polymeric fibers and films with a high modulus has been extensively investigated and interesting results have been obtained for polyethyelne. ${ }^{1-5}$ This research is based on the assumption that the theoretical Young's modulus could be realized if the chains are fully aligned and extended and if the specimen is almost completely crystalline. In an attempt to prepare such an ideal sample, chain alignment induced by deformation or flow has been extensively investigated. Recently Matsuo et al. $^{5}$ produced ultradrawn polyethylene films whose Young's modulus at $20^{\circ} \mathrm{C}$ was $216 \mathrm{GPa}$, which is nearly equal to the crystal lattice modulus. Crystallinity was slightly higher than $97 \%$. In this specimen, the second order orientation factor of the $c$-axes was almost unity and the birefringence was beyond $60 \times 10^{-3} \cdot 6,7$ The orientation of amorphous chain segments has been determined from birefringence data obtained by

\footnotetext{
$\dagger$ To whom correspondence should be addressed.
}

subtraction of the crystalline contribution from the total birefringence, assuming sample additivity as indicated in the following equation $^{8}$

$$
\Delta_{\text {total }}=X_{\mathrm{c}} \Delta_{\mathrm{c}}+\left(1-X_{\mathrm{c}}\right) \Delta_{\mathrm{a}}+\Delta_{\mathrm{f}}
$$

where $\Delta_{\text {total }}$ is the total birefringence of the bulk specimen, $\Delta_{\mathrm{c}}$ is the crystalline birefringence, $\Delta_{\mathrm{a}}$ is the amorphous birefringence, and $\Delta_{\mathrm{f}}$ is the form birefringence. In eq $1, \Delta_{\mathrm{c}}$ and $\Delta_{\mathrm{a}}$ are given by

$$
\Delta_{\mathrm{c}}=\Delta_{\mathrm{c}}^{0} F_{200}, \quad \Delta_{\mathrm{a}}=\Delta_{\mathrm{a}}^{0} F_{200}^{a}
$$

where $\Delta_{\mathrm{c}}^{0}$ and $\Delta_{\mathrm{a}}^{0}$ are the intrinsic birefringence of the crystalline and amorphous phases, respectively.

Interestingly, it has been reported that total birefringence is beyond $60 \times 10^{-36,7}$ and this is higher than the intrinsic birefringence $\Delta_{\mathrm{c}}^{0}$, $58.5 \times 10^{-3}$, calculated from the three principal refractive indices of crystals of the $n$-paraffin 
$\mathrm{C}_{36} \mathrm{H}_{74}$ evaluated by using the bond-polarizability values given by Bunn and Daubeny, ${ }^{9}$ which is the reverse of what is normally observed. According to these authors, the refractive indices $n_{\mathrm{a}}, n_{\mathrm{b}}$, and $n_{\mathrm{c}}$ were 1.514 , 1.519 , and 1.575 , respectively. The birefringence, $\Delta_{\mathrm{c}}^{0}$, given by $n_{\mathrm{c}}-\left(n_{\mathrm{a}}+n_{\mathrm{b}}\right) / 2$, is thus equal to $58.5 \times 10^{-3}$. The defect of such treatment is due to the fact that the principal refractive indices were estimated only by assuming the atomic arrangements within the crystal unit and neglecting the uncertain effects of the internal field. Furthermore, form birefringence has been generally neglected on estimating total birefringence.

This paper is focused on the form birefringence of ultradrawn polyethylene films to explain the value of total birefringence higher than the intrinsic crystal birefringence using the two dimensional model, in which oriented crystalline layers are surrounded by anisotropic amorphous phases.

\section{THEORY}

Let the composite structural unit be as shown in Figure 1, in which oriented crystallites are surrounded by amorphous phases. Diagram (b) represents the system containing voids. In contrast, diagram (a) represents the system obtained by removing voids from diagram (b).

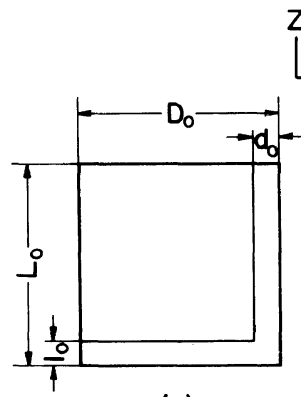

(a)

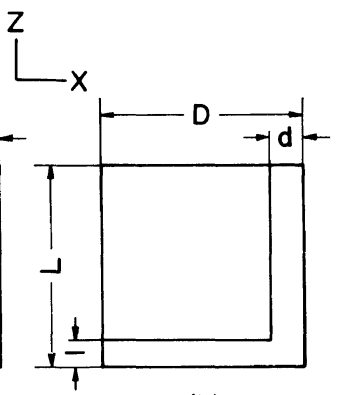

(b)
Figure 1. Composite structural unit of crystalline polymer whose crystallites are surrounded by amorphous phases.

(a) without void; (b) containing voids.
Thus, there exists no void in the system. In both systems, amorphous layers are adjacent to oriented crystalline layers with the interfaces perpendicular to the $x$ and $z$ axes. In diagram (a), fractions of length correspond to $l_{0} / L_{0}$ in the $z$ direction and $d_{0} / D_{0}$ in the $x$ direction, while in diagram (b), the fraction corresponds to be $l / L$ and $d / D$ in the $z$ and $x$ directions, respectively. It is well known, of course, that at $d_{0} / D_{0}(=d / D)=0$, these models correspond to a series model, while at $l_{0} / L_{0}(=l / L)=0$, they correspond to a parallel model. Assuming that there is no void in the crystalline phase, we have

$$
\left(D_{0}-d_{0}\right)\left(L_{0}-l_{0}\right)=(D-d)(L-l)
$$

Thus, the crystallinity $X_{\mathrm{c}}$ is defined by

$$
X_{\mathrm{c}}=\frac{\left(L_{0}-l_{0}\right)\left(D_{0}-d_{0}\right)}{L_{0} D_{0}}=\left(1-\frac{l_{0}}{L_{0}}\right)\left(1-\frac{d_{0}}{D_{0}}\right)(4)
$$

As discussed above, this assumes that the voids exist only in the amorphous phase. Thus, volume fraction, $\phi_{\mathrm{s}}$, of voids within the amorphous phase may be given by

$$
\begin{aligned}
\phi_{\mathrm{s}} & =1-\frac{l_{0} D_{0}+d_{0}\left(L_{0}-l_{0}\right)}{l D+d(L-l)} \\
& =1-\left\{1-\left(1-\frac{l_{0}}{D_{0}}\right)\left(1-\frac{d_{0}}{D_{0}}\right)\right\} / \\
& \left\{\left(\frac{L}{L_{0}}\right)\left(\frac{D}{D_{0}}\right)-X_{\mathrm{c}}\right\}
\end{aligned}
$$

Generally, a refractive index $n$ is given by

$$
n^{2}=\varepsilon \mu
$$

where $\varepsilon$ is a dielectric constant and $\mu$ is a magnetic permeability. Except for ferroelectrics, $\mu$ is almost equal to unity and eq 6 reduces to

$$
n^{2}=\varepsilon
$$

Define $\varepsilon_{c z}$ and $\varepsilon_{c x}$ to be dielectric constants of the crystal phase in the $z$ and $x$ directions, respectively, and $\varepsilon_{a z}$ and $\varepsilon_{a x}$ to be dielectric constants of the amorphous phase in the $z$ and 
$x$ directions, respectively. Following Cvikl et al. ${ }^{10}$ electric constants $\varepsilon_{z}$ and $\varepsilon_{x}$ of the bulk specimen in the $z$ and $x$ directions in diagram (a) may be given by

$$
\begin{aligned}
\frac{1}{\varepsilon_{z}}= & \frac{\left(l_{0} / L_{0}\right)}{\varepsilon_{a z}}+\left(1-\frac{l_{0}}{L_{0}}\right) / \\
& \left\{\frac{d_{0}}{D_{0}} \varepsilon_{a z}+\left(1-\frac{d_{0}}{D_{0}}\right) \varepsilon_{c z}\right\}
\end{aligned}
$$

and

$$
\begin{aligned}
\frac{1}{\varepsilon_{x}}= & \frac{\left(d_{0} / D_{0}\right)}{\varepsilon_{a x}}+\left(1-\frac{d_{0}}{D_{0}}\right) / \\
& \left\{\frac{l_{0}}{L_{0}} \varepsilon_{a x}+\left(1-\frac{l_{0}}{L_{0}}\right) \varepsilon_{c x}\right\}
\end{aligned}
$$

where $\varepsilon_{c z}$ and $\varepsilon_{c x}$ are dielectric constants in the crystal phase. In the uniaxial orientational mode,

$$
\begin{aligned}
\sqrt{\varepsilon_{c z}}= & \frac{2}{3}\left(\sqrt{\varepsilon_{c}^{c}}-\sqrt{\varepsilon_{c}^{a}}\right) F_{200}^{c} \\
& +\frac{2}{3}\left(\sqrt{\varepsilon_{c}^{b}}-\sqrt{\varepsilon_{c}^{a}}\right) F_{200}^{b} \\
& +\frac{1}{3}\left(\sqrt{\varepsilon_{c}^{c}}+\sqrt{\varepsilon_{c}^{b}}+\sqrt{\varepsilon_{c}^{a}}\right)
\end{aligned}
$$

and

$$
\begin{aligned}
\sqrt{\varepsilon_{c x}}= & \frac{1}{3}\left(\sqrt{\varepsilon_{c}^{c}}+\sqrt{\varepsilon_{c}^{b}}+\sqrt{\varepsilon_{c}^{a}}\right) \\
& -\frac{1}{3}\left(\sqrt{\varepsilon_{c}^{c}}-\sqrt{\varepsilon_{c}^{a}}\right) F_{200}^{c} \\
& -\frac{1}{3}\left(\sqrt{\varepsilon_{c}^{b}}-\sqrt{\varepsilon_{c}^{a}}\right) F_{200}^{b}
\end{aligned}
$$

where $F_{200}^{c}$ and $F_{200}^{b}$ are the second order orientation factors of the $c$ and $b$ axes determined by X-ray diffraction measurements and $\varepsilon_{c}^{c}, \varepsilon_{b}^{c}$, and $\varepsilon_{a}^{c}$ are dielectric constants in the directions of the $c-, b$-, and $a$-axes, respectively. Similarly, dielectric constants $\varepsilon_{a z}$ and $\varepsilon_{a x}$ of the amorphous phase are given by

$$
\sqrt{\varepsilon_{a z}}=\frac{2}{3}\left(\sqrt{\varepsilon_{\|}^{a}}-\sqrt{\varepsilon_{\perp}^{a}}\right) F_{200}^{a m}+\frac{1}{3}\left(\sqrt{\varepsilon_{\|}^{a}}+2 \sqrt{\varepsilon_{\perp}^{a}}\right)
$$

and

$$
\left.\sqrt{\varepsilon_{a x}}=\frac{1}{3} \sqrt{\varepsilon_{\|}^{a}}+2 \sqrt{\varepsilon_{\perp}^{a}}\right)-\frac{1}{3}\left(\sqrt{\varepsilon_{\|}^{a}}-\sqrt{\varepsilon_{\perp}^{a}}\right) F_{200}^{a m}
$$

where $F_{200}^{a m}$ denotes the second order orientation factor of amorphous chain segments and $\varepsilon_{\|}^{a}$ and $\varepsilon_{\perp}^{a}$ are dielectric constants of the intrinsic amorphous phases parallel and perpendicular to the chain axis.

In diagram (b), dielectric constants $\varepsilon_{z}^{\prime}$ and $\varepsilon_{x}^{\prime}$ of the bulk specimen in the $z$ and $x$ directions may be given by

$$
\begin{aligned}
\frac{1}{\varepsilon_{z}^{\prime}}= & \frac{1}{\varepsilon_{a z}^{\prime}}\left\{1-\left(1-\frac{d_{0}}{D_{0}}\right)\left(\frac{D_{0}}{D}\right)\right\} \\
& +\left(1-\frac{l_{0}}{L_{0}}\right)\left(\frac{L_{0}}{L}\right) / \\
& {\left[\left\{1-\left(1-\frac{d_{0}}{D_{0}}\right)\left(\frac{D_{0}}{D}\right) \varepsilon_{a z}^{\prime}\right.\right.} \\
& \left.+\left(1-\frac{d_{0}}{D_{0}}\right)\left(\frac{D_{0}}{D}\right) \varepsilon_{c z}\right]
\end{aligned}
$$

and

$$
\begin{aligned}
\frac{1}{\varepsilon_{x}^{\prime}}= & \frac{1}{\varepsilon_{a x}^{\prime}}\left\{1-\left(1-\frac{d_{0}}{D_{0}}\right)\left(\frac{D_{0}}{D}\right)\right\} \\
& +\left(1-\frac{d_{0}}{D_{0}}\right)\left(\frac{D_{0}}{D}\right) / \\
& {\left[\left\{1-\left(1-\frac{l_{0}}{L_{0}}\right)\left(\frac{L_{0}}{L}\right) \varepsilon_{a x}^{\prime}\right.\right.} \\
& \left.+\left(1+\frac{l_{0}}{L_{0}}\right)\left(\frac{L_{0}}{L}\right) \varepsilon_{c x}\right]
\end{aligned}
$$

where $\varepsilon_{a z}^{\prime}$ and $\varepsilon_{a x}^{\prime}$ correspond to dielectric constants of amorphous phase containing voids and are given by

$$
\varepsilon_{a z}^{\prime}=\varepsilon_{a z}\left(1-\phi_{\mathrm{s}}\right)+\varepsilon_{\mathrm{s}} \phi_{\mathrm{s}}
$$


and

$$
\varepsilon_{a x}^{\prime}=\varepsilon_{a x}\left(1-\phi_{\mathrm{s}}\right)+\varepsilon_{\mathrm{s}} \phi_{\mathrm{s}}
$$

where $\varepsilon_{\mathrm{s}}$ is a dielectric constant of voids. If the specimen is immersed in liquid, $\varepsilon_{\mathrm{s}}$ corresponds to the dielectric constant of the liquid.

Instead of eq 16 and 17, the dielectric constants of amorphous phase containing voids can be also defined, respectively, as follows:

$$
\frac{1}{\varepsilon_{a z}^{\prime}}=\frac{1-\phi_{\mathrm{s}}}{\varepsilon_{a z}}+\frac{\phi_{\mathrm{s}}}{\varepsilon_{\mathrm{s}}}
$$

and

$$
\frac{1}{\varepsilon_{a x}^{\prime}}=\frac{1-\phi_{\mathrm{s}}}{\varepsilon_{a x}}+\frac{\phi_{\mathrm{s}}}{\varepsilon_{\mathrm{s}}}
$$

When the crystal chain axes are oriented perfectly with respect to the stretching direction, eq 10 and 11 reduce to

$$
\sqrt{\varepsilon_{c z}}=\sqrt{\varepsilon_{c}^{c}}
$$

and

$$
\sqrt{\varepsilon_{c x}}=\frac{1}{2}\left(\sqrt{\varepsilon_{c}^{a}}+\sqrt{\varepsilon_{c}^{b}}\right)
$$

The optical anisotropy of amorphous chains can be given by an ellipsoid of rotation about the chain axis. In actual calculation, two kinds of orientational behaviour are assumed; perfect orientation of amorphous chain segments and random orientation. In the former, eq 12 and 13 reduce to

$$
\sqrt{\varepsilon_{a z}}=\sqrt{\varepsilon_{\|}^{a}}
$$

and

$$
\sqrt{\varepsilon_{a x}}=\sqrt{\varepsilon_{\perp}^{a}}
$$

In the latter, we have

$$
\sqrt{\varepsilon_{a z}}=\sqrt{\varepsilon_{a x}}=\frac{1}{3}\left(\sqrt{\varepsilon_{\|}^{a}}+2 \sqrt{\varepsilon_{\perp}^{a}}\right)
$$

In numerical calculations, $\sqrt{\varepsilon_{\|}^{a}}$ and $\sqrt{\varepsilon_{\perp}^{a}}$ were obtained assuming the following simple relation $^{11}$;

$$
\sqrt{\varepsilon_{\|}^{a}}=\sqrt{\varepsilon_{c}^{c}}\left(\frac{\rho_{a}}{\rho_{c}}\right)
$$

and

$$
\sqrt{\varepsilon_{\perp}^{a}}=\frac{1}{2}\left(\sqrt{\varepsilon_{c}^{a}}+\sqrt{\varepsilon_{c}^{b}}\right)\left(\frac{\rho_{a}}{\rho_{c}}\right)
$$

where $\rho_{c}$ and $\rho_{a}$ are the densities of the crystal and amorphous phases, respectively. The total birefringences $\Delta$ and $\Delta^{\prime}$ in diagrams (a) and (b) may be defined by

$$
\Delta=n_{z}-n_{x}=\sqrt{\varepsilon_{z}}-\sqrt{\varepsilon_{x}}
$$

and

$$
\Delta^{\prime}=n_{z}^{\prime}-n_{x}^{\prime}=\sqrt{\varepsilon_{z}^{\prime}}-\sqrt{\varepsilon_{x}^{\prime}}
$$

Thus, form birefringence may be given by

$$
\Delta_{\text {form }}=\Delta^{\prime}-\Delta
$$

A series of calculations show that eq 29 can be used to calculate total birefringence, $\Delta_{\text {total }}$, is a very complicated form even in the simple two dimensional model and the simple additivity shown in eq 1 is only an assumption.

\section{RESULTS AND DISCUSSION}

To represent the morphology of ultradrawn polyethylene, volume crystallinity, $X_{c}$, is fixed at $0.97(97 \%)$. The values of $\left(1-l_{0} / L_{0}\right)$ and $\left(1-d_{0} / D_{0}\right)$ must be set to assure $X_{c}=0.97$ in eq 4. Three cases are used and each set of parameters is listed in Table I. In Case I, parallel coupling between amorphous and crystal phases is predominant, while in Case II, series coupling is predominant. In Case III,

Table I. Parameter sets to calculate birefringence

\begin{tabular}{rccc}
\hline & Case I & Case II & Case III \\
\hline $1-\frac{l_{0}}{L_{0}}$ & 0.9999 & 0.9700 & 0.9800 \\
\hline $1-\frac{d_{0}}{D_{0}}$ & 0.9700 & 0.9999 & 0.9898
\end{tabular}




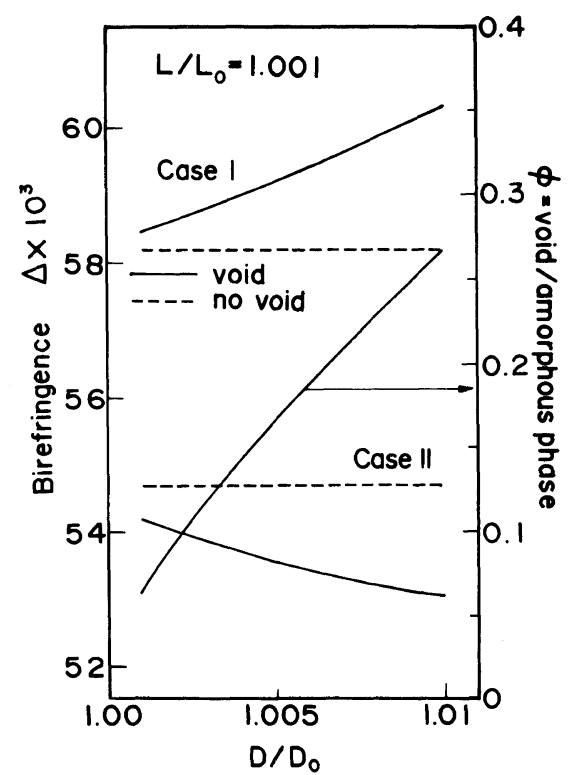

Figure 2. Birefringence against $D / D_{0}$ at $L / L_{0}=1.001$.

crystal phases are surrounded by thin amorphous phases.

Figure 2 shows birefringence with increasing $D / D_{0}$ when $L / L_{0}$ is fixed at 1.001 . In Case I, corresponding to parallel coupling, the birefringence in this system containing no void is about $58.25 \times 10^{-3}$ which is close to the value of the intrinsic crystalline birefringence. On the other hand, in Case II, corresponding to series coupling, the value is about $54.7 \times 10^{-3}$. This indicates that birefringence is very sensitive to morphology even in ultradrawn films with crystallinity of $97 \%$.

With increasing $D / D_{0}$, i.e., volume fraction, $\phi_{\mathrm{s}}$, birefringence in Case I increases. The increase in voids in Case I is shown in Model A in Figure 3. In this model system, voids exist only in the amorphous region, $d L$, showing parallel coupling and no void allows to exist in the very narrow amorphous region, $l D$. Here it may be noted that at $D / D_{0}>1.085$ (i.e., $\phi>0.24)$, birefringence is beyond $60 \times 10^{-3}$. This has been reported for ultradrawn polyethylene films. ${ }^{6,7}$ In contrast, birefringence in Case II decreases with increasing voids. The increase in voids in Case II can be represent-
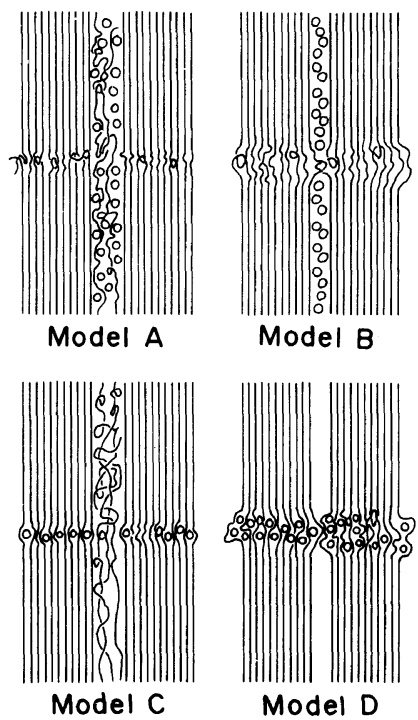

Figure 3. Models representing morphological properties.

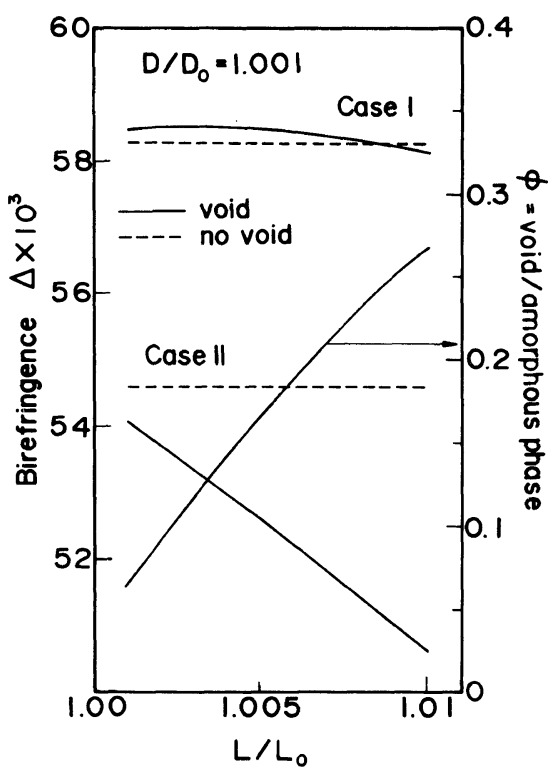

Figure 4. Birefringence against $L / L_{0}$ at $D / D_{0}=1.001$.

ed as Model B in Figure 3. In this model system, voids exist only in the very small amorphous phase, $d L$, showing parallel coupling. This model was proposed to image morphology such that voids exist at the narrow amorphous boundary between crystallites parallel 
in the stretching direction.

Figure 4 shows birefringence with increasing $L / L_{0}$ at $D / D_{0}=1.001$. Under this condition, the existence of a number of voids is not realistic in Case I, since it is impossible to occupy a large space of voids in small amorphous phase, $d L$ geometrically, as shown Model $\mathrm{C}$ in Figure 3. It is seen that birefringence becomes slightly lower with increasing $L / L_{0}$. In Case II, voids exist only in the amorphous phase, $l D$, indicating the series coupling as shown in Model D in Figure 3. This is reasonable in terms of geometrical arrangement. Birefringence is lower than the real value in the absence of voids and becomes lower with increasing voids.

Figure 5 shows birefringence with increasing $L / L_{0}$ and $D / D_{0}$ in Case III. This was proposed to clarify change in birefringence, when voids exist in the amorphous phases which surround the crystalline layers. The value decreases with increasing voids. The decreasing degree is as in-between the decreasing Case I and Case II.

Through a series of results in Figures 2, 4, and 5 , it turns out that birefringence with voids is sensitive to the morphology of ultradrawn

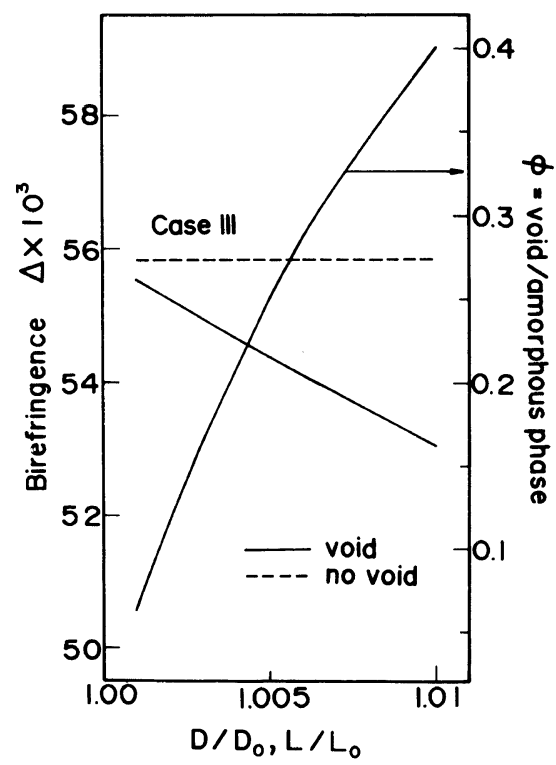

Figure 5. Birefringence against $L / L_{0}$ and $D / D_{0}$. polyethylene. If the parallel approximation is more appropriate than the series model, the birefringence becomes more pronounced as the content of voids increases. Actually, as birefringence in Case $I$ is higher than the intrinsic crystal birefringence at $\phi_{\mathrm{s}}>0.24$.

According to Bettelheim and Stein, ${ }^{12}$ the birefringence of polyethylene film exhibited a minimum value when plotted against the refractive index of the solvent in the manner predicted by Wiener ${ }^{13}$ for form birefringence of particles elongated in the stretching direction. They reported that the curvature of birefringence against refractive index of swelling liquid increases with the draw ratio, owing to the increase in the particle elongation and/or orientation. They pointed out that the contribution from form birefringence at the minimum of these curves becomes zero according to the Wiener theory. ${ }^{13}$ A question arises as to whether the concept by Wiener can be applied to the system such as ultradrawn films whose the $c$-axes are oriented perfectly with respect to the stretching direction and volume crystallinity is beyond $97 \%$. That is, whether there is no contribution from form birefringence at the minimum (or maximum)

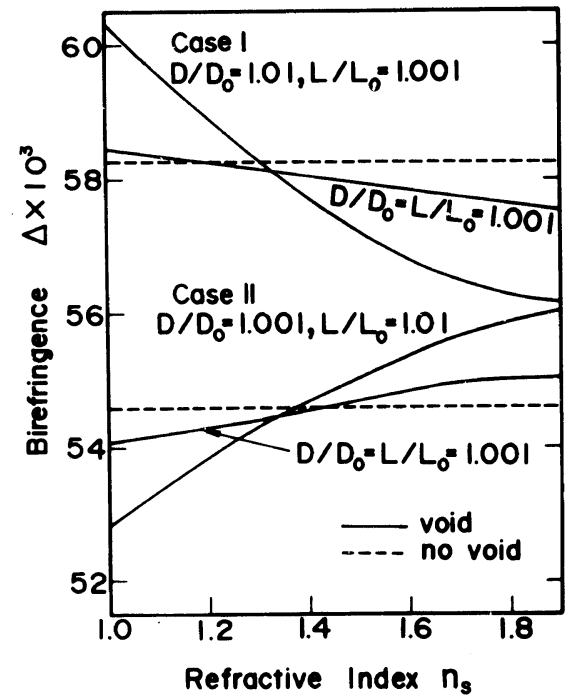

Figure 6. Birefringence against refractive index $n_{\mathrm{s}}$ of the swelling liquid. 
of measurable birefringence against the refractive index of solvent.

Figure 6 shows variation of birefringence calculated as a function of refractive index $n_{\mathrm{s}}$ of the swelling liquid in the indicated cases. In Case I, birefringence decreases with $n_{\mathrm{s}}$ and this is pronounced with increasing $D / D_{0}$ at $L / L_{0}=1.001$. The curve at $D / D_{0}=1.01$ shows a minimum value with further increase of $n_{\mathrm{s}}$ beyond 1.9 and again increases. In contrast, birefringence in Case II increases with $n_{\mathrm{s}}$ and this becomes pronounced with $L / L_{0}$ at a fixed value of $L / L_{0} \quad(=1.001)$. Thus, when the morphological properties of a test specimen can be appropriated with a parallel model, the apparent birefringence become less pronounced with increasing $n_{\mathrm{s}}$ in the given index range. In contrast, if the series approximation is more appropriate than the parallel model, apparent birefringence becomes more pronounced.

Here it may be noted that a very difficult problem arises when obtaining the real birefringence with no contribution from form birefringence by using the method proposed by Wiener ${ }^{13}$ because the minimum or maximum point of birefringence curve is not equal to the real birefringence, (dotted curve). Furthermore, birefringence calculated by assuming a perfect orientation of amorphous chain segments was almost equal to that calculated by assuming random orientation as shown in Figures 2, 4-6. This is attributed to high crystallinity such as $97 \%$. That is, the contribution from amorphous phase is not sensitive enough to determine the birefringence of a bulk specimen. This indicates that the second order orientation factor of the amorphous chain segments within ultradrawn films cannot be determined from birefringence data as estimated by subtraction of crystalline contribution from the total birefringence.

All numerical results calculated from eq 18 and 19 gave almost same values as those calculated from eq 16 and 17 . Thus, the composite mode of voids and amorphous phase is almost independent of value of total birefringence of ultradrawn polyethylene.

\section{REFERENCES}

1. P. Smith and P. J. Lemstra, J. Mater. Sci., 15, 505 (1980).

2. P. Smith, P. J. Lemstra, J. P. L. Pijpers, and A. M. Kiel, Colloid Polym. Sci., 258, 1070 (1980).

3. K. Furuhata, K. Yokokawa, and K. Miyasaka, J. Polym. Sci., Polym. Phys. Ed., 22, 133 (1984).

4. T. Kanamoto, A. Tsuruta, K. Tanaka, and R. S. Porter, Polym. J., 15, 327 (1983).

5. M. Matsuo and C. Sawatari, Macromolecules, 19, 2036 (1986)

6. M. Matsuo and C. Sawatari, Macromolecules, 21, 1653 (1988).

7. C. Sawatari and M. Matsuo, Polymer, 30, 1603 (1989).

8. R. S. Stein and F. H. Norris, J. Polym. Sci., 21, 381 (1956).

9. C. W. Bunn and R. de Daubeny, Trans. Faraday Soc., 50, 1173 (1954).

10. B. Cvikl, D. Mori, and W. Franklin, Mol. Cryst. Liquid Cryst., 12, 267 (1971).

11. N. Sakaguchi, T. Oda, A. Nakai, and H. Kawai, Sen- $i$ Gakkaishi, 33, 499 (1977).

12. F. A. Bettelheim and R. S. Stein, J. Polym. Sci., 27, 567 (1958).

13. O. Wiener, Abh. Sachs. Akad. d. Wiss. Math. Phys. Kl., 32, 507 (1912). 